\title{
Automated Electron Tomography Software for High -Precision, 3-D Reconstructions
}

T. Oikawa*, H. Nishioka*, H. Furukawa**, M. Shimizu**, Y. Suzuki** and B. L. Armbruster***

* JEOL Ltd., Akishima, Tokyo 196-8558, Japan

** JEOL System Technology Ltd., Akishima, Tokyo 196-8558, Japan

*** JEOL USA, Peabody, MA 01960

Electron tomographic techniques are essential for modeling three-dimensional mass densities of biological structures in the size range of $10-500 \mathrm{~nm}$. Generating three-dimensional reconstructions from serial sections is a widely accepted technique [1], but this methodology requires extensive specimen preparation and data processing to merge feature sets from each section. The accuracy of measurement in the Z-direction due to material lost during the sectioning process and section shrinkage from beam damage increases the margin of error.

The recently developed Computerized Tomography (CT) reconstruction method [2] obtains 3 dimensional information from a tilt series. With complete tilt series datacollection, the ideal resolution range of $2-5 \mathrm{~nm}$, similar to that obtained by means of the more laborintensive serial section reconstruction method, could be achieved. New modifications to the software include automatic image shift correction, an algorithm for interpolating missing wedge information, and automatic identification of the tilt axis.

Figure 1 is a schematic representation of the 3-dimensional information acquisition and reconstruction process. Images may be acquired on photographic film, imaging plates or on a CCD digital camera. A separate computer is maintained off-line for 3-D reconstruction calculations. The software is PC-based with a simple operation panel (Fig. 2). After entering the name of the image data file and the tilt angle on the screen, the program starts the reconstruction calculation.

The CT reconstruction method has been applied to the study of several biological and materials science specimens. One example is shown in Figure 3, a reconstruction of a desmosome from conventionally embedded, sectioned cells. The images were obtained by tilting the specimen from -60 to +60 degrees with 2.5 degree steps in a JEM-1230 operating at $120 \mathrm{kV}$, though it is possible to tilt $+/-80$ degrees with the cryopolepiece. The images were recorded by means of a Gatan BioScan camera. Phase boundaries in reconstructions of gyroid (polystyreneblockpolyisoprene-diblock-copolymer) are easily examined in the interactive 3-D volume display.

\section{References}

[1] T. Ehara et al., J. Elect. Microsc. 45 (1996) 159.

[2] H. Furukawa et al., JEOL News 36E (1) (2001) 50. 


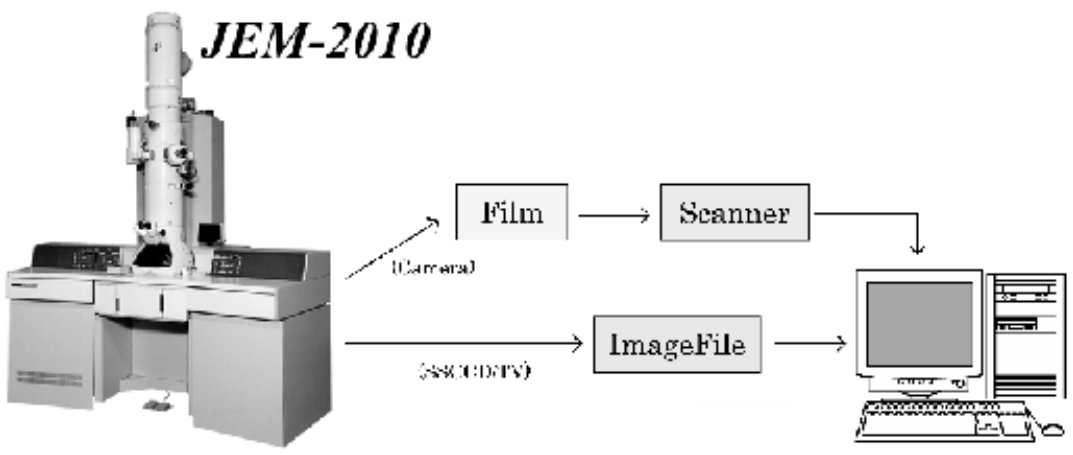

FIG. 1. Schematic diagram of 3-dimensional information acquisition and reconstruction process.

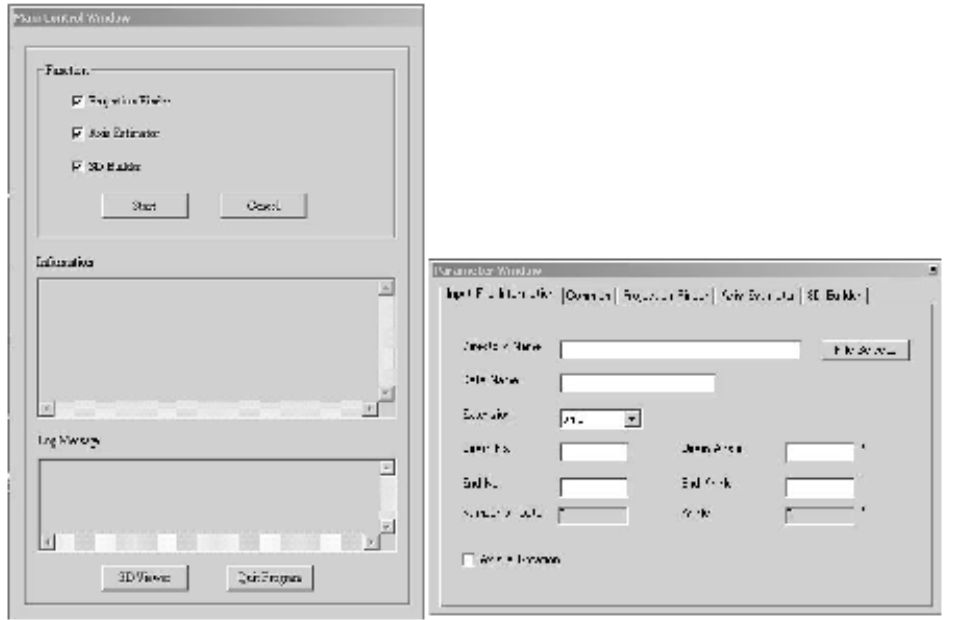

FIG. 2. GUI operation panel screens for CT reconstruction method.

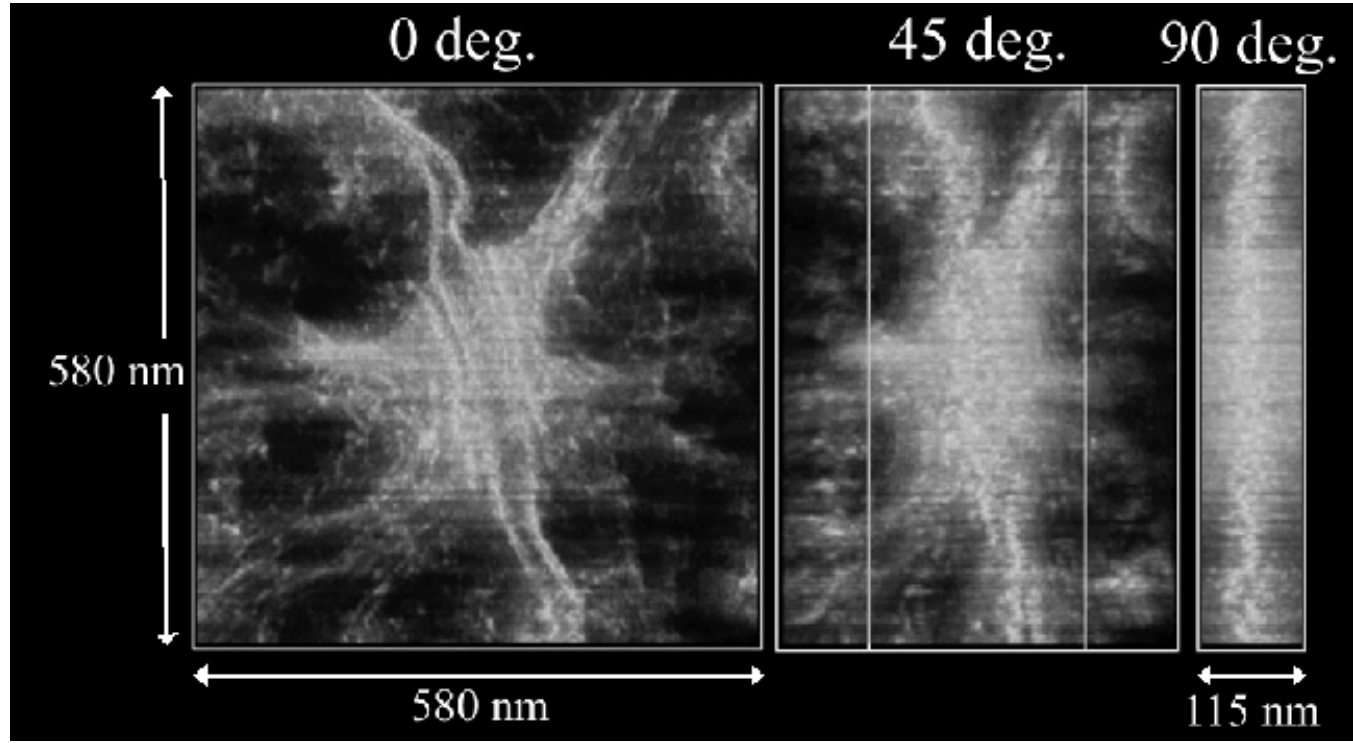

FIG. 3. Reconstruction of a section of a conventionally embedded desmosome. Digital images were combined from a -60 to +60 degree tilt series with 2.5 degree steps. 